\title{
Diversity of wood - decaying fungi in Haikou Forestry Farm, Yunnan Province, P.R. China
}

\author{
He X, Chen JZ and Zhao CL*
}

College of Biodiversity Conservation, Southwest Forestry University, Kunming 650224, P.R. China

He X, Chen JZ, Zhao CL 2021 - Diversity of wood-decaying fungi in Haikou Forestry Farm, Yunnan Province, P.R. China. Studies in Fungi 6(1), 365-377, Doi 10.5943/sif/6/1/27

\begin{abstract}
Nine field trips carried out in Haikou Forestry Farm, Yunnan Province, P.R. China resulted in 681 specimens of wood-decaying fungi. The present paper summarizes 52 species collected that are distributed in 37 genera, 16 families, 6 orders including their hosts and substrates. A checklist of wood-decaying fungi in Haikou Forestry Farm is also given. Phylogenetic analysis of ITS nrRNA gene region was performed for all the collected samples with maximum likelihood, maximum parsimony and Bayesian inference methods. The phylogenetic tree showed that fifty-two species nested in sixteen families belonging to six orders in Agaricomycetes.
\end{abstract}

Keywords - Basidiomycota - biological resources - diversity - Haikou Forestry Farm - mycota

\section{Introduction}

The diversity for flora of seed plants in Yunnan Province, P.R. China is observably high, and the endemic species of woody plants are rich, in which both supply good substrates for wooddecaying fungi. Wood-decaying fungi are kind of large basidiomycetes that grow on various kinds of wood, such as the living trees, dead standing trees, fallen trunk, fallen branch and stump (Dai 2012a), which can be used for industrial value, medicinal value, edible value and economic value (Russell \& Paterson 2006, Dai et al. 2015, Vinay et al. 2015, M'Barek et al. 2020, Wu et al. 2020, Runnel et al. 2021).

Haikou Forestry Farm is located in Haikou Town, Kunming, belonging to the Jinsha River system and the geographical location is between $102^{\circ} 28^{\prime}-102^{\circ} 38^{\prime} \mathrm{E}$ and $24^{\circ} 4^{\prime}-24^{\circ} 56^{\prime} \mathrm{N}$, with the altitude of 1800-2400 $\mathrm{m}$ (Xu et al. 2015, Zhang et al. 2017). It has a subtropical monsoon climate and the native tree species with mostly subtropical evergreen broadleaf trees (Xiong \& Zhou 2019). The main vegetation includes warm coniferous forest (Pinus yunnanensis Franch., P. armandii Franch. and Keteleeria evelyniana Mast.), deciduous broad-leaved forest (Alnus nepalensis D. Don), and semi-humid evergreen broad-leaved forest (Lithocarpus dealbatus (J. D. Hooker et Thomson ex Miquel) Rehder and Castanopsis delavayi Franch.).

However, the previously documented wood-decaying fungi are mostly in northwest Yunnan Province, China, and few polypore and corticioid fungi have been reported in here so far. According to the modern taxonomy (Dai 2012a), wood-decaying fungi mainly belong to ten orders of Agaricomycetes, viz., Agaricales, Auriculariales, Cantharellales, Corticiales, Gloeophyllales, Hymenochaetales, Polyporales, Russulales, Thelephorales and Trechisporales. Therefore, the current wood-decaying fungi catalogues include poroid and corticioid hymenophore. In the present study, nine field trips were carried out in different areas of Haikou Forestry Farm, and about 681 specimens were collected, in which 52 species belonging to 37 genera, 16 families, 6 orders, which 
were identified from these materials. This paper is going to summarize the distribution of wooddecaying fungi and enrich the fungal diversity in this area.

\section{Materials \& Methods}

\section{Morphological studies}

The studied specimens are deposited at the herbarium of Southwest Forestry University (SWFC), Kunming, Yunnan Province, P.R. China. Macromorphological descriptions were based on field notes. Colour terms are from Petersen (1996). Micromorphological data were obtained from the dried specimens, and observed under a light microscope following Dai (2012a). The following abbreviations were used for the micro characteristics description: $\mathrm{KOH}=5 \%$ potassium hydroxide, $\mathrm{CB}=$ Cotton Blue, $\mathrm{CB}-=$ acyanophilous, $\mathrm{CB}+=$ cyanophilous, $\mathrm{IKI}=$ Melzer's reagent, $\mathrm{IKI}-=$ both inamyloid and indextrinoid, $\mathrm{L}=$ mean spore length (arithmetic average of all spores), $\mathrm{W}=$ mean spore width (arithmetic average of all spores), $Q=$ variation in the $\mathrm{L} / \mathrm{W}$ ratios between the specimens studied, $n(a / b)=$ number of spores (a) measured from given number $(b)$ of specimens.

\section{Molecular procedures and phylogenetic analysis}

CTAB rapid plant genome extraction kit-DN14 (Aidlab Biotechnologies Co., Ltd, Beijing) was used to obtain genomic DNA from dried specimens, according to the manufacturer's instructions that a small piece of dried fungal specimen (about $30 \mathrm{mg}$ ) was ground to powder with liquid nitrogen. The powder was transferred to a $1.5 \mathrm{~mL}$ centrifuge tube, suspended in $0.4 \mathrm{~mL}$ of lysis buffer, and incubated in a $65^{\circ} \mathrm{C}$ water bath for $60 \mathrm{~min}$. After that, $0.4 \mathrm{~mL}$ phenol-chloroform (24:1) was added to each tube and the suspension was shaken vigorously. After centrifugation at $13,000 \mathrm{rpm}$ for $5 \mathrm{~min}, 0.3 \mathrm{~mL}$ supernatant was transferred to a new tube and mixed with $0.45 \mathrm{~mL}$ binding buffer. The mixture was then transferred to an adsorbing column (AC) for centrifugation at 13,000 rpm for $0.5 \mathrm{~min}$. Then, $0.5 \mathrm{~mL}$ inhibitor removal fluid was added in AC for a centrifugation at $12,000 \mathrm{rpm}$ for $0.5 \mathrm{~min}$. After washing twice with $0.5 \mathrm{~mL}$ washing buffer, the AC was transferred to a clean centrifuge tube, and $100 \mathrm{~mL}$ elution buffer was added to the middle of adsorbed film to elute the genome DNA. ITS region was amplified with primer pair ITS5 and ITS4 (White et al. 1990). The PCR procedure for ITS was as follows: initial denaturation at $95^{\circ} \mathrm{C}$ for 3 min, followed by 35 cycles at $94^{\circ} \mathrm{C}$ for $40 \mathrm{~s}, 58^{\circ} \mathrm{C}$ for $45 \mathrm{~s}$ and $72^{\circ} \mathrm{C}$ for $1 \mathrm{~min}$, and a final extension of $72^{\circ} \mathrm{C}$ for $10 \mathrm{~min}$. The PCR products were purified and directly sequenced at Kunming Tsingke Biological Technology Limited Company, Kunming Yunnan Province, P.R. China. All newly generated sequences were deposited at GenBank (Table 1).

Sequencher 4.6 (GeneCodes, Ann Arbor, MI, USA) was used to edit the DNA sequence. Sequences were aligned in MAFFT 7 (http://mafft.cbrc.jp/alignment/server/) using the "G-INS-i" strategy and manually adjusted in BioEdit (Hall 1999). Sequences of Tremella flava Chee J. Chen and T. mesenterica Retz. obtained from GenBank was used as an outgroup to root tree following He et al. (2019) in ITS analysis (Fig. 1).

Maximum parsimony analysis was applied to the ITS dataset sequences. Approaches to phylogenetic analysis followed Zhao \& Wu (2017) and the tree construction procedure was performed in PAUP* version 4.0b10 (Swofford 2002). All characters were equally weighted and gaps were treated as missing data. Trees were inferred using the heuristic search option with TBR branch swapping and 1000 random sequence additions. Max-trees were set to 5000, branches of zero length were collapsed and all parsimonious trees were saved. Clade robustness was assessed using a bootstrap (BT) analysis with 1,000 replicates (Felsenstein 1985). Descriptive tree statistics tree length (TL), consistency index (CI), retention index (RI), rescaled consistency index (RC), and homoplasy index (HI) were calculated for each Maximum Parsimonious Tree (MPT) generated. Sequences were also analyzed using Maximum Likelihood (ML) with RAxML-HPC2 through the Cipres Science Gateway (www.phylo.org; Miller et al. 2009). Branch support (BS) for ML analysis was determined by 1000 bootstrap replicates. 
Table 1 Names, sample numbers and corresponding GenBank accession numbers of ITS sequences used in this study

\begin{tabular}{|c|c|c|c|}
\hline Species name & Sample no. & $\begin{array}{l}\text { GenBank } \\
\text { accession no. }\end{array}$ & References \\
\hline Acanthofungus rimosus & $\mathrm{Wu} 9601-1$ & MF043521 & He et al. (2019) \\
\hline Acanthophysellum cerussatum & He 2208 & KX306874 & He et al. (2019) \\
\hline Aleurobotrys botryosus & He 2712 & KX306877 & He et al. (2019) \\
\hline Antrodia tanakae & CLZhao 720 & MG231457 & This study \\
\hline Armillaria aotearoa & NZFS 2425 & NR151846 & Hood \& Ramsfield 2016 \\
\hline Auricularia angiospermarum & BJFC 017274 & NR151847 & Wu et al. 2015 \\
\hline Auricularia villosula & CLZhao 1296 & MG231464 & This study \\
\hline Bankera fuligineoalba & REB-285 & JN135196 & He et al. (2019) \\
\hline Boletinellus merulioides & $2630 \mathrm{a}$ & KM248952 & He et al. (2019) \\
\hline Bondarzewia berkeleyi & Dai 12759 & KJ583202 & He et al. (2019) \\
\hline Boreostereum radiatum & RLG-9717-Sp & HM536085 & Garcia-Sandoval et al. 2011 \\
\hline Brunneoporus malicola & CLZhao 1530 & MG231451 & This study \\
\hline Candelabrochaete langloisii & FP-110343 & KY948793 & He et al. (2019) \\
\hline Ceraceomyces serpens & HHB-15692-Sp & KP135031 & He et al. (2019) \\
\hline Cinereomyces lindbladii & CBS 290.71 & MH860129 & Vu et al. 2019 \\
\hline Cinereomyces lindbladii & CLZhao 1523 & MG231489 & This study \\
\hline Coniolepiota spongodes & ECV-2010a & HM488756 & He et al. (2019) \\
\hline Coprinellus curtus & SZMC-NL-2339 & FM878016 & He et al. (2019) \\
\hline Coprinus comatus & AFTOL-ID 626 & AY854066 & He et al. (2019) \\
\hline Coriolopsis polyzona & Cui 11040 & KR605824 & He et al. (2019) \\
\hline Crepatura ellipsospora & CLZhao 697 & MK343695 & Ma \& Zhao 2019 \\
\hline Cyclomyces lamellatus & Cui 7629 & JQ279603 & He et al. (2019) \\
\hline Cylindrobasidium laeve & CLZhao 767 & MG231497 & This study \\
\hline Daedalea quercina & FFUI-4 & MN596945 & Direct Submission \\
\hline Daedaleopsis confragosa & CLZhao 1481 & MG231506 & This study \\
\hline Efibula americana & FP-102165 & KP135016 & He et al. (2019) \\
\hline Eichleriella alliciens & HHB 7194 & KX262120 & He et al. (2019) \\
\hline Exidiopsis effusa & OM 19136 & KX262145 & He et al. (2019) \\
\hline Flammulina velutipes & AFTOL-ID 558 & AY854073 & He et al. (2019) \\
\hline Fomitiporia mediterranea & AFTOL-ID 688 & AY854080 & He et al. (2019) \\
\hline Funalia gallica & CLZhao 1306 & MG231491 & This study \\
\hline Funalia trogii & CLZhao 1557 & MG231874 & This study \\
\hline Gloeophyllum sepiarium & CLZhao 732 & MG231532 & This study \\
\hline Gloeophyllum trabeum & 1320 & HM536094 & He et al. (2019) \\
\hline Gloeoporus taxicola & CLZhao 1441 & MG231549 & This study \\
\hline Grifola frondosa & AFTOL-ID 701 & AY854084 & He et al. (2019) \\
\hline Gymnopus confluens & ZRL 20151148 & LT716054 & He et al. (2019) \\
\hline Heliocybe sulcata & IBUG 9930 & HM536095 & He et al. (2019) \\
\hline Heterobasidion annosum & $06129 / 6$ & KJ583211 & He et al. (2019) \\
\hline Heterobasidion insulare & CLZhao 2899 & MK268944 & This study \\
\hline Heterobasidion orientale & CLZhao 696 & MG231561 & This study \\
\hline Hymenochaetopsis yasudae & CLZhao 1422 & MG231607 & This study \\
\hline Hymenopellis radicata & AFTOL-ID 561 & DQ241780 & He et al. (2019) \\
\hline Hyphoderma macaronesicum & TFC Mic 15939 & NR119817 & Schoch et al. 2014 \\
\hline Hyphoderma setigerum & CBS 421.72 & MH860512 & Vu et al. 2019 \\
\hline Hyphoderma transiens & CLZhao 1365 & MK404378 & This study \\
\hline Hyphoderma variolosum & CBS 735.91 & MH862321 & Vu et al. 2019 \\
\hline Hyphodermella rosae & FP-150552 & KP134978 & He et al. (2019) \\
\hline Inocutis dryophilus & DLL 2012-001 & KU139186 & He et al. (2019) \\
\hline Irpex lacteus & CLZhao 1258 & MG231709 & This study \\
\hline Junghuhnia crustacea & X 262 & JN710553 & Miettinen et al. 2012 \\
\hline Lacrymaria lacrymabunda & CBS 211.31 & MH855192 & He et al. (2019) \\
\hline Laurilia sulcata & CBS 365.49 & MH856552 & Vu et al. 2019 \\
\hline Lenzitopsis daii & Yuan 2959 & JN169799 & He et al. (2019) \\
\hline
\end{tabular}


Table 1 Continued.

\begin{tabular}{|c|c|c|c|}
\hline Species name & Sample no. & $\begin{array}{l}\text { GenBank } \\
\text { accession no. }\end{array}$ & References \\
\hline Lycoperdon ericaeum & ZRL 20151498 & LT716030 & He et al. (2019) \\
\hline Macrolepiota dolichaula & xml 2013058 & LT716021 & He et al. (2019) \\
\hline Megalocystidium luridum & CBS 106.71 & MH860024 & Vu et al. 2019 \\
\hline $\begin{array}{l}\text { Megasporoporiella } \\
\text { subcavernulosa }\end{array}$ & CLZhao 1438 & MG231737 & This study \\
\hline Melanogaster rivularis & S 190 & HQ714731 & He et al. (2019) \\
\hline Meripilus giganteus & FP-135344 & KP135307 & He et al. (2019) \\
\hline Meruliopsis albostramineus & HHB-10729 & KP135051 & He et al. (2019) \\
\hline Microporus xanthopus & CLZhao 1285 & MG231749 & This study \\
\hline Nigroporus vinosus & KA17-0261 & MN294801 & Direct Submission \\
\hline Oligoporus farinosus & CIEFAP 91 & JX090117 & Pildain \& Rajchenberg 2013 \\
\hline Omphalotus olearius & AFTOL-ID 1718 & DQ494681 & He et al. (2019) \\
\hline Perenniporia hainaniana & Cui 6364 & JQ861743 & He et al. (2019) \\
\hline Phaeophlebiopsis caribbeana & HHB-6990 & KP135415 & He et al. (2019) \\
\hline Phanerochaete concrescens & CLZhao 1430 & MG231768 & This study \\
\hline Phanerochaete sordida & CLZhao 1459 & MG231774 & This study \\
\hline Phellinus ellipsoideus & Cui 4270 & JQ837948 & He et al. (2019) \\
\hline Phlebiopsis crassa & CLZhao 786 & MG231791 & This study \\
\hline Phylloporus pelletieri & Pp 1 & DQ534566 & He et al. (2019) \\
\hline Pisolithus tinctorius & AWW 219 & EU718114 & He et al. (2019) \\
\hline Polyozellus multiplex & AFTOL-ID 677 & DQ411528 & He et al. (2019) \\
\hline Polyporus arcularius & CLZhao 1338 & MG231798 & This study \\
\hline Porphyrellus porphyrosporus & MB 97-023 & DQ534563 & He et al. (2019) \\
\hline Postia lactea & Cui 9319 & KX900894 & Direct Submission \\
\hline Psathyrella candolleana & ZRL 20151400 & LT716063 & He et al. (2019) \\
\hline Pseudochaete subrigidula & He 1157 & JQ716403 & He et al. (2019) \\
\hline Pycnoporus sanguineus & ZRL 2015009 & LT716078 & He et al. (2019) \\
\hline Rhizochaete americana & FP-102188 & KP135409 & He et al. (2019) \\
\hline Rhizomarasmius oreinus & AQUI 6763 & NR132910 & Moreau et al. 2015 \\
\hline Rhodocollybia maculata & AFTOL-ID 540 & DQ404383 & He et al. (2019) \\
\hline Rhodotus asperior & HKAS 56754 & KC179737 & He et al. (2019) \\
\hline Rigidoporus undatus & Miettinen 13591 & KY948731 & He et al. (2019) \\
\hline Sarcodon joeides & REB-270 & KC571772 & He et al. (2019) \\
\hline Schizophyllum commune & CLZhao 1528 & MG231811 & This study \\
\hline Schizophyllum leprieurii & ROBLEDO 1313 & KM098065 & Direct Submission \\
\hline Scleroderma areolatum & AWW 211 & EU718115 & He et al. (2019) \\
\hline Sparsitubus nelumbiformis & Cui 8497 & KX880631 & He et al. (2019) \\
\hline Steccherinum bourdotii & CLZhao 1347 & MG231820 & This study \\
\hline Steccherinum ochraceum & CLZhao 2897 & MK269280 & This study \\
\hline Stereum hirsutum & CLZhao 1411 & MG231830 & This study \\
\hline Stereum rugosum & CLZhao 1310 & MG231836 & This study \\
\hline Stereum sanguinolentum & CLZhao 668 & MG231838 & This study \\
\hline Trametes hirsuta & CLZhao 1544 & MG231868 & This study \\
\hline Trametopsis cervina & TJV $93216 \mathrm{~T}$ & JN165020 & He et al. (2019) \\
\hline Tremella flava & CBS 8471 & KY105681 & He et al. (2019) \\
\hline Tremella mesenterica & CBS 6973 & NR155937 & He et al. (2019) \\
\hline Trulla dentipora & AS 2288 & KY970064 & Direct Submission \\
\hline Veluticeps fimbriata & L-10628-Sp & HM536100 & He et al. (2019) \\
\hline Xylobolus frustulatus & He 2231 & KU881905 & He et al. (2019) \\
\hline
\end{tabular}

MrModeltest 2.3 (Nylander 2004) was used to determine the best-fit evolution model for each data set for Bayesian inference (BI). Bayesian inference was calculated with MrBayes3.1.2 with a general time reversible $(\mathrm{GTR}+\mathrm{I}+\mathrm{G})$ model of DNA substitution and a gamma distribution rate variation across sites (Ronquist \& Huelsenbeck 2003). Four Markov chains were run for 2 runs 
from random starting trees for 1500 thousand generations (Fig. 1), and trees were sampled every 100 generations. The first one-fourth generations were discarded as burn-in. A majority rule consensus tree of all remaining trees was calculated. A majority rule consensus tree of all remaining trees was calculated. Branches were considered as significantly supported if they received maximum likelihood bootstrap $(\mathrm{BS})>70 \%$, maximum parsimony bootstrap $(\mathrm{BT})>50 \%$, or Bayesian posterior probabilities (BPP) $>0.95$.

\section{Results}

\section{Molecular phylogeny}

The ITS dataset (Fig. 1) included sequences from 102 fungal specimens representing 101 species. The dataset had an aligned length of 1389 characters, of which 308 characters were constant, 283 parsimony-uninformative, and 798 parsimony-informative. Maximum parsimony analysis yielded 1 equally parsimonious tree $(\mathrm{TL}=8644, \mathrm{CI}=0.2678, \mathrm{HI}=0.7322, \mathrm{RI}=0.4535$, $\mathrm{RC}=0.1215)$. The best-fit model for ITS alignment estimated and applied in the Bayesian was $\mathrm{GTR}+\mathrm{I}+\mathrm{G}$, lset $\mathrm{nst}=6$, rates $=$ invgamma; prset statefreqpr $=$ dirichlet $(1,1,1,1)$. Bayesian resulted in a similar topology with an average standard deviation of split frequencies $=0.029534(\mathrm{BI})$, and the effective sample size (ESS) across the two runs is the double of the average ESS (avg ESS) $=$ 354.

The phylogeny (Fig. 1) inferred from ITS sequences demonstrated that fifty-two species nested in sixteen families, Auriculariaceae, Bondarzewiaceae, Dacryobolaceae, Fomitopsidaceae, Gelatoporiaceae, Gloeophyllaceae, Hymenochaetaceae, Hyphodermataceae, Irpicaceae, Phanerochaetaceae, Physalacriaceae, Polyporaceae, Schizophyllaceae, Schizoporaceae, Steccherinaceae and Stereaceae, belonging to six orders Agaricales, Auriculariales, Gloeophyllales, Hymenochaetales, Polyporales, Russulales in Agaricomycetes.

\section{Checklist}

An alphabetical list (according to genus name) of wood-decaying fungi identified in these investigations is given below. The authors of scientific names are according to the second edition of Authors of Fungal Names (http://www.indexfungorum.org/AuthorsOfFungalNames.html). Substrate and collecting data are provided after the name of each species. The hosts are listed alphabetically, and within the same host tree, they are arranged by the order: living tree, dead standing tree, trunk, fallen branch and stump. The collectors and collection numbers are listed alphabetically, too (Dai 2011, 2012a).

1. Antrodia tanakae (Murrill) Spirin \& Miettinen, on the fallen branch of Acacia dealbata Link, CLZhao 720; on the stump of Acacia dealbata, CLZhao 1536

2. Auricularia villosula Malysheva, on the fallen branch of Alnus nepalensis, CLZhao 1428; on the trunk of Juglans regia L., CLZhao 743; on the trunk of Quercus, CLZhao 1296; on the fallen branch of Quercus, CLZhao 1340

3. Basidioradulum crustosum (Pers.) Zmitr., Malysheva \& Spirin, on the fallen angiosperm branch, CLZhao 3028

4. Bjerkandera adusta (Willd.) P. Karst, on the stump of Pinus yunnanensis, CLZhao 1555; on the dead tree of Quercus, CLZhao 1275

5. Brunneoporus malicola (Berk. \& M.A. Curtis) Audet, on the stump of Acacia dealbata, CLZhao 1524; on the trunk of Quercus acutissima Carr., CLZhao 1530

6. Byssomerulius corium (Pers.) Parmasto, on the fallen angiosperm branch, CLZhao 1560; on the fallen branch of Pinus yunnanensis, CLZhao 734, CLZhao 781; on the fallen branch of Quercus, CLZhao 693, CLZhao 1266, CLZhao 1274, CLZhao 1313; on the stump of Quercus, CLZhao 1341

7. Cinereomyces lindbladii (Berk.) Jülich, on the trunk of Pinus yunnanensis, CLZhao 1523

8. Crepatura ellipsospora C.L. Zhao, on the trunk of Alnus, CLZhao 697; on the fallen branch of Quercus, CLZhao 868, CLZhao 1260, CLZhao 1265 
9. Cylindrobasidium laeve (Pers.) Chamuris, on the dead bamboo, CLZhao 756, CLZhao 767

10. Daedaleopsis confragosa (Bolton) J. Schröt, on the trunk of Alnus nepalensis, CLZhao 1481

11. Funalia gallica (Fr.) Bondartsev \& Singer, on the trunk of Alnus nepalensis, CLZhao 1309; on the fallen branch of Alnus nepalensis, CLZhao 1306

12. Funalia trogii Berk., on the trunk of Alnus nepalensis, CLZhao 1540; on the angiosperm trunk, CLZhao 741, CLZhao 3009, CLZhao 1557; on the fallen angiosperm branch CLZhao 1552

13. Fuscoporia torulosa (Pers.) T. Wagner \& M. Fisch, on the fallen branch of Quercus, CLZhao 1305

14. Gloeophyllum sepiarium (Wulfen) P. Karst, on the trunk of Pinus yunnanensis, CLZhao 732, CLZhao 764; on the fallen branch of Pinus yunnanensis, CLZhao 774, CLZhao 904; on the stump of Pinus yunnanensis, CLZhao 731, CLZhao 784

15. Gloeoporus dichrous (Fr.) Bres., on the fallen branch of Alnus nepalensis, CLZhao 1471

16. Gloeoporus taxicola (Pers.) Gilb. \& Ryvarden, on the trunk of Pinus armandii, CLZhao 1441

17. Heterobasidion insulare (Murrill) Ryvarden, on the stump of Pinus yunnanensis, CLZhao 2899

18. Heterobasidion orientale Tokuda, T. Hatt. \& Y.C. Dai, trunk of Pinus yunnanensis, CLZhao 696

19. Hymenochaete adusta (Lév.) Har. \& Pat., J. Bot, on the trunk of Alnus, CLZhao 700

20. Hymenochaete rheicolor (Mont.) Lév., on the trunk of Alnus, CLZhao 672, CLZhao 679; on the fallen branch of Quercus, CLZhao 666, CLZhao 671, CLZhao 682

21. Hymenochaete villosa (Lév.) Bres., on the trunk of Quercus acutissima, CLZhao 1533

22. Hymenochaetopsis corrugata (Fr.) S.H. He \& Jiao Yang, on the fallen angiosperm branch, CLZhao 2893

23. Hymenochaetopsis yasudae (Imazeki) S.H. He \& Jiao Yang, on the fallen branch of Alnus nepalensis, CLZhao 1422, CLZhao 1445; on the living tree of Pinus armandii, CLZhao 1475; on the fallen branch of Pinus armandii, CLZhao 1486, CLZhao 1495, CLZhao 1549

24. Hyphoderma transiens (Bres.) Parmasto, on the fallen angiosperm branch, CLZhao 1493; on the trunk of Populus yunnanensis Dode, CLZhao 1365

25. Hyphodontia tropica Sheng $\mathrm{H}$. Wu, on the fallen angiosperm branch, CLZhao 2898, CLZhao 2901

26. Irpex lacteus (Fr.) Fr., on the trunk of Acacia dealbata, CLZhao 1258

27. Lopharia cinerascens (Schwein.) G. Cunn., on the fallen branch of Alnus nepalensis, CLZhao 1499

28. Megasporoporiella subcavernulosa (Y.C. Dai \& Sheng H. Wu) B.K. Cui, on the fallen branch of Alnus nepalensis, CLZhao 1438, CLZhao 1466; on the stump of Alnus nepalensis, CLZhao 1412; on the fallen angiosperm branch, CLZhao 2966, CLZhao 2984, CLZhao 3016; on the stump of Cupressus funebris Endl., CLZhao 1444, CLZhao 1491

29. Microporus xanthopus (Fr.) Kuntze, on the fallen branch of Alnus nepalensis, CLZhao 1503; on the fallen angiosperm branch, CLZhao 3012; on the trunk of Quercus, CLZhao 1253, CLZhao 1285; on the fallen branch of Quercus, CLZhao 1268, CLZhao 1304; on the stump of Quercus, CLZhao 1343

30. Phanerochaete concrescens Spirin \& Volobuev, on the fallen branch of Alnus nepalensis, CLZhao 1541, CLZhao 1545; on the fallen angiosperm branch, CLZhao 2929, CLZhao 2931, CLZhao 2939, CLZhao 2940, CLZhao 2945, CLZhao 2946, CLZhao 2949; on the trunk of Pinus yunnanensis, CLZhao 2916

31. Phanerochaete sordida (P. Karst.) J. Erikss. \& Ryvarden, on the trunk of Alnus, CLZhao 698; on the fallen branch of Alnus nepalensis, CLZhao 1459, CLZhao 1541, CLZhao 1545; on the angiosperm trunk, CLZhao 1461; on the fallen angiosperm branch, CLZhao 2929, CLZhao 2931, CLZhao 2939, CLZhao 2940, CLZhao 2945, CLZhao 2946, CLZhao 2949, CLZhao 4738, CLZhao 4746, CLZhao 4754; on the fallen branch of Pinus armandii, CLZhao 1515; on the trunk of Pinus yunnanensis, CLZhao 2916

32. Phellinus gilvus (Schwein.) Pat., on the fallen branch of Alnus nepalensis, CLZhao 1334 
33. Phlebiopsis crassa (Lév.) Floudas \& Hibbett, on the angiosperm trunk, CLZhao 786; on the fallen branch of Coriaria nepalensis Wall., CLZhao 1295, CLZhao 1308; on the trunk of Pinus yunnanensis, CLZhao 724; on the trunk of Quercus, CLZhao 1269, CLZhao 1314; on the fallen branch of Quercus acutissima, CLZhao 1532

34. Polyporus arcularius (Batsch) Fr. on the stump of Coriaria nepalensis, CLZhao 1338; on the fallen branch of Quercus, CLZhao 1316

35. Postia hibernica (Berk. Broome) Jülich, on the fallen angiosperm branch, CLZhao 2903; on the fallen branch of Pinus yunnanensis, CLZhao 2909

36. Pulcherricium coeruleum (Lam.) Parmasto, on the fallen branch of Alnus nepalensis, CLZhao 1434; on the fallen angiosperm branch, CLZhao 3003, CLZhao 3020

37. Schizophyllum commune Fr., on the trunk of Acacia dealbata, CLZhao 1527; on the fallen branch of Acacia dealbata, CLZhao 1528, CLZhao 1529; on the trunk of Alnus nepalensis, CLZhao 1537; on the stump of Alnus nepalensis, CLZhao 1562; on the stump of Eucalyptus robusta, CLZhao 1561

38. Steccherinum bourdotii Saliba \& A. David, on the fallen branch of Alnus nepalensis, CLZhao 1472; on the fallen branch of Pinus armandii, CLZhao 1347

39. Steccherinum ochraceum (Pers. ex J.F. Gmel.) Gray, on the fallen angiosperm branch, CLZhao 2897, CLZhao 2968

40. Stereum gausapatum (Fr.) Fr., on the trunk of Alder, 11 January 2017, CLZhao 668, CLZhao 669, CLZhao 673, CLZhao 677, CLZhao 683, CLZhao 694, CLZhao 707; on the dead tree of Quercus, 22 April 2017, CLZhao 1259, CLZhao 1270, CLZhao 1290, CLZhao 1300, CLZhao 1318, CLZhao 1320

41. Stereum hirsutum (Willd.) Pers., on the fallen branch of Alnus nepalensis, CLZhao 1404, CLZhao 1405, CLZhao 1427, CLZhao 1455, CLZhao 1457, CLZhao 1465, CLZhao 1469, CLZhao 1470, CLZhao 1479, CLZhao 1482, CLZhao 1489, CLZhao 1498; on the fallen angiosperm branch, CLZhao 740; on fallen branch of Coriaria nepalensis, CLZhao 1559; on fallen branch of Pinus yunnanensis, CLZhao 2906; on the fallen branch of Quercus, CLZhao 1291

42. Stereum rugosum Pers., on the trunk of Quercus, CLZhao 1310

43. Stereum sanguinolentum (Alb. \& Schwein.) Fr., on the trunk of Alnus, CLZhao 673; on the stump of Pinus yunnanensis, CLZhao 669

44. Trametes hirsuta (Wulfen) Lloyd, on the fallen branch of Alnus nepalensis, CLZhao 1544; on the angiosperm trunk, CLZhao 1344; on the trunk of Pinus yunnanensis, CLZhao 739

45. Trametes strumosa (Fr.) Zmitr., on the angiosperm trunk, CLZhao 718

46. Trametes versicolor (L.) Lloyd, on the trunk of Alnus nepalensis, CLZhao 1539, CLZhao 1546; on the fallen branch of Alnus nepalensis, CLZhao 1431; on the stump of Alnus nepalensis, CLZhao 1510; on the angiosperm trunk, CLZhao 748, CLZhao 1293, CLZhao 1477; on the trunk of Cupressus funebris, CLZhao 1509; on the trunk of Quercus, CLZhao 1330; on the fallen branch of Quercus, CLZhao 686, CLZhao 714, CLZhao 1302, CLZhao 1307

47. Trametopsis cervina (Schwein.) Tomšovský, on the trunk of Alnus nepalensis, CLZhao 1246; on the fallen branch of Quercus, CLZhao 1315

48. Trichaptum abietinum (Dicks.) Ryvarden, on the angiosperm stump, CLZhao 719, CLZhao 723, CLZhao 736, CLZhao 777, CLZhao 3004; on the trunk of Pinus yunnanensis, CLZhao 730

49. Tubulicrinis xantha C.L. Zhao, on the fallen branch of Pinus yunnanensis, CLZhao 2868, CLZhao 2869, CLZhao 2883

50. Xylodon kunmingensis L. W. Zhou \& C.L. Zhao, on the fallen angiosperm branch, CLZhao 3010, CLZhao 3019; on the stump of angiosperm, CLZhao 755; on the fallen branch of Pinus yunnanensis, CLZhao 752

51. Xylodon nespori (Bres.) Hjortstam \& Ryvarden, on the trunk of Cupressus funebris, CLZhao 1492

52. Xylodon rimosissimus (Peck) Hjortstam \& Ryvarden, on the fallen branch of Pinus armandii, CLZhao 1487 


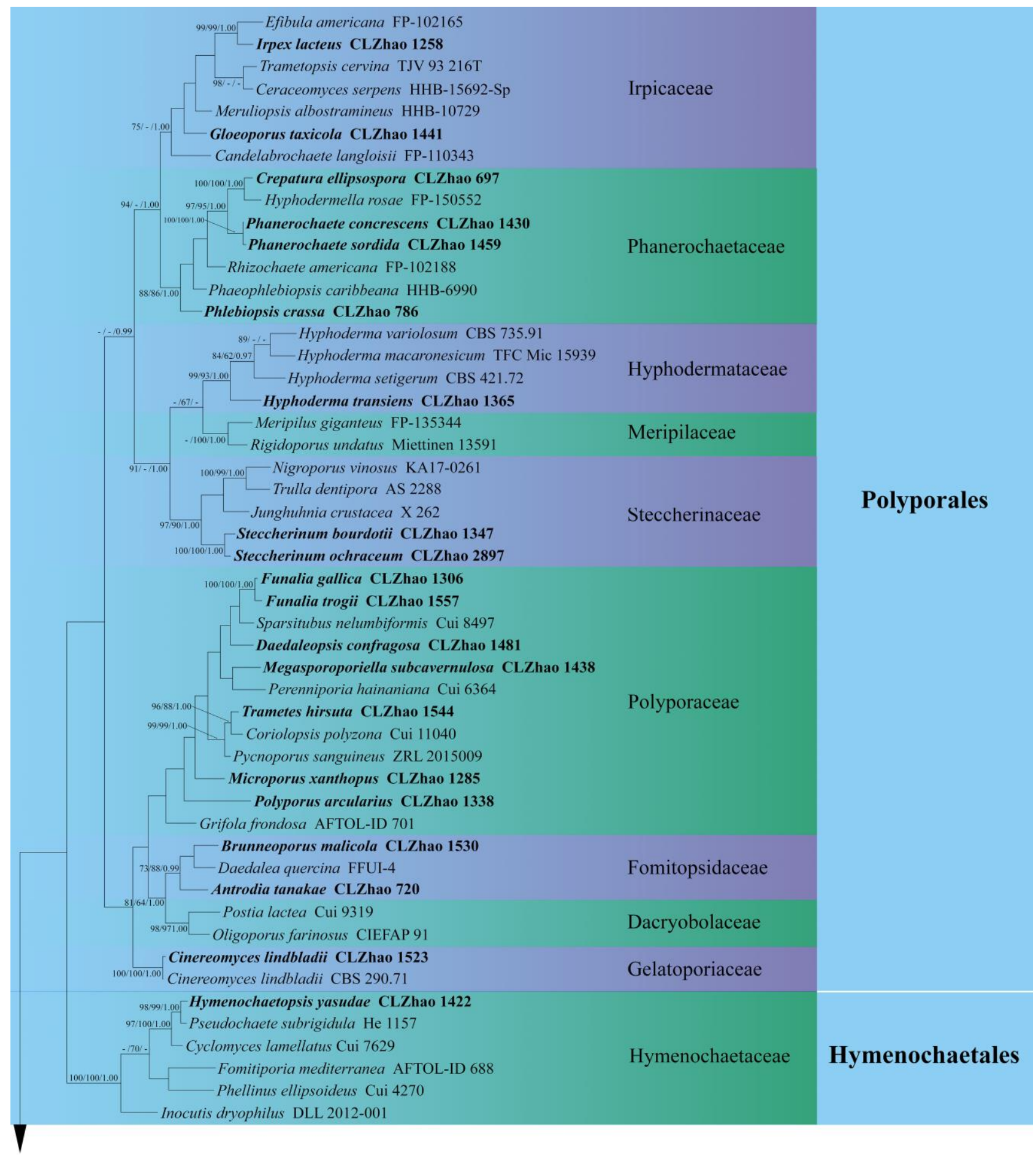

Fig. 1 - Maximum parsimony strict consensus tree illustrating the phylogeny of 52 species with related taxa in Agaricomycetes based on ITS sequences. Branches are labelled with maximum likelihood bootstrap equal to or greater than $70 \%$, parsimony bootstrap equal to or greater than $50 \%$ and Bayesian posterior probabilities equal to or greater than 0.97 , respectively. The taxa from the present study are indicated in black bold. 


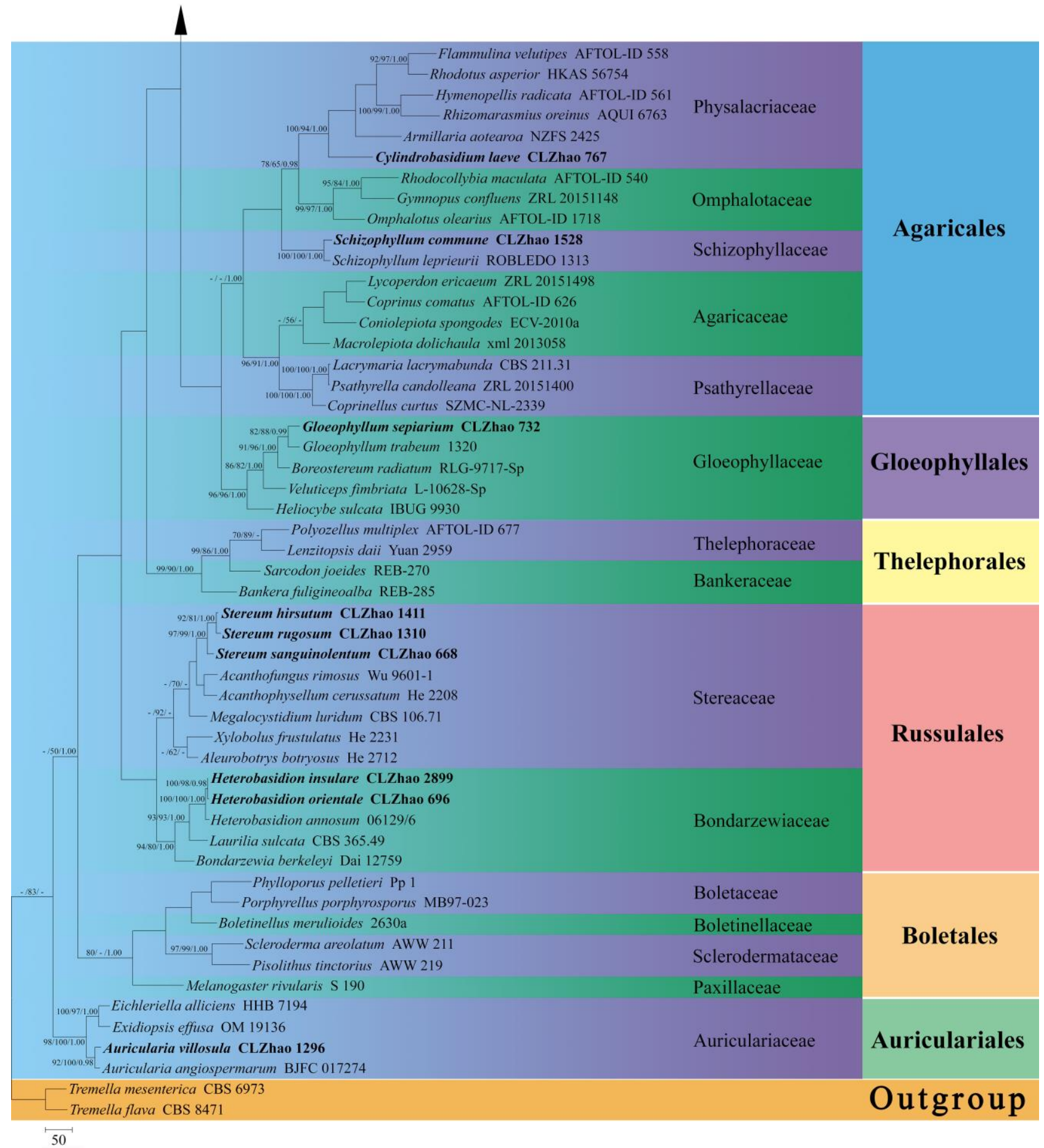

Fig. 1 - Continued.

\section{Discussion}

In the kingdom Fungi, the phyla Ascomycota and Basidiomycota cover around $97 \%$ of all fungal species (Willis 2018). According to the latest version of Ainsworth \& Bisby's Dictionary of the Fungi (Kirk et al. 2008), there are 1589 genera and more than 30 thousand species of Basidiomycota, which comprise nearly $32 \%$ of all described fungal taxa (Dai et al. 2015), and more and more taxa were recorded worldwide every year, which observably increase the fungal diversity (Sarma \& Hyde 2018, Yafetto 2018, Freitas-Neto et al. 2019, Hipol et al. 2019, Ayesha et al. 2020, De Leon AM et al. 2020, Huang et al. 2020, Himani \& Krishnappa 2020, Blanco-Dios 2021, Kumar et al. 2021, Wang \& Zhao 2021, Zong et al. 2021). 
Although some notable explorations of wood-decaying fungi have been made from Yunnan Province (Cui \& Dai 2012, Wu et al. 2017, Zhao \& Wu 2017, Shen et al. 2018, Wu et al. 2018, Liu et al. 2019, Luo et al. 2019, Xu et al. 2019, Chen \& Zhao 2020, Huang et al. 2020, Wang et al. 2020, Wu et al. 2021, Zong et al. 2021), only three new species were found from Haikou Forestry Farm (Ma \& Zhao 2019, Shi et al. 2019, He et al. 2020). Species in the present list are mostly new to the studied area. The 681 specimens belong to 52 species, which are distributed in 37 genera, 16 families, 6 orders, in which 11 are pathogenic (Dai 2012b), and 28 are medicinal (Dai \& Yang 2008), and a few are edible (Dai et al. 2010). Among them, 28 species belong to order Polyporales and 14 species belong to order Hymenochaetales and 6 species belong to order Russulales, and 2 species belong to order Agaricales and 1 species belong to order Auriculariales and 1 species belong to order Gloeophyllales.

The molecular phylogenetic analyses with combined nLSU, SSU, 5.8s, rpb1, rpb2, and tef1 datasets for the subphyla Agaricomycotina, Pucciniomycotina and Ustilaginomycotina revealed that 1928 currently used genera names are distributed in 241 families, 68 orders, 18 classes and four subphyla (He et al. 2019). In the present study, 52 species nested in 37 genera, 16 families, 6 orders based on ITS dataset, which is similar to the topology of the phylogenetic analyses in He et al. (2019).

This work will comprehensively improve the understanding of the diversity of wooddecaying fungi in this area, which is conducive to the rational utilization and effective protection of fungal resources, and provides scientific basis for the prevention and control of forest diseases in this farm.

\section{Acknowledgements}

The researches were supported by the Yunnan Fundamental Research Project (Grant No. 202001AS070043) and the Science Foundation of Southwest Forestry University (Project No. 111715).

\section{References}

Ayesha P, Maham A, Ariza A, Husnain H. 2020 - "Ganoderma lucidum sensu lato" - a sacred mushroom for immortality. Studies in Fungi 5(1), 508-516. Doi 10.5943/sif/5/1/31

Blanco-Dios JB. 2021 - Notes on the genus Entoloma s.l. in the Northwest of the Iberian Peninsula (XI): a new species in subgenus Entoloma. Studies in Fungi 6(1), 64-70. Doi $10.5943 / \mathrm{sif} / 6 / 1 / 2$

Chen JZ, Zhao CL. 2020 - Morphological and molecular identification of four new resupinate species of Lyomyces (Hymenochaetales) from southern China. MycoKeys 65, 101-118. Doi 10.3897/mycokeys.65.48660

Cui BK, Dai YC. 2012 - Wood-decaying fungi in eastern Himalayas 3. Polypores from Laojunshan Mountains, Yunnan Province. Mycosystema 31(4), 486-492.

Dai YC. 2011 - A revised checklist of corticioid and hydnoid fungi in China for 2010. Mycoscience 52, 69-79. Doi: 10.1007/S10267-010-0068-1

Dai YC. 2012a - Polypore diversity in China with an annotated checklist of Chinese polypores. Mycoscience 53, 49-80. Doi 10.1007/s10267-011-0134-3

Dai YC. 2012b - Pathogenic wood-decaying fungi on woody plants in China. Mycosystema 31(4), 493-509.

Dai YC, Cui BK, Si J, He SH et al. 2015 - Dynamics of the worldwide number of fungi with emphasis on fungal diversity in China. Mycological Progress 14, 62.

Doi 10.1007/s11557-015-1084-5

Dai YC, Yang ZL. 2008 - A revised checklist of medicinal fungi in China. Mycosystema 27(6), 801-824. Doi 10.3969/j.issn.1672-6472.2008.06.001

Dai YC, Zhou LW, Yang ZL, Wen HA et al. 2010 - A revised checklist of edible fungi in China. Mycosystema 29(1), 1-21. 
De Leon AM, Dulay AR, Villanueva AL, Kalaw SP. 2020 - Optimal culture conditions and toxicity assessment of Fomitopsis feei (Fr.): a newly documented macro fungus from Philippines. Studies in Fungi 5(1), 491-507. Doi 10.5943/sif/5/1/30

Felsenstein J. 1985 - Confidence intervals on phylogenetics: an approach using bootstrap. Evolution 39, 783-791. Doi 10.1111/j.1558-5646.1985.tb00420.x

Freitas-Neto JF, Sousa JO, Ovrebo CL, Baseia IG. 2019 - Geastrum echinulatum and G. rusticum (Geastraceae, Basidiomycota) - two new records for Central America. Studies in Fungi 4(1), 14-20. Doi 10.5943/sif/4/1/2

Garcia-Sandoval R, Wang Z, Binder M, Hibbett DS. 2011 - Molecular phylogenetics of the Gloeophyllales and relative ages of clades of Agaricomycotina producing a brown rot. Mycologia 103(3), 510-524. Doi 10.3852/10-209

Hall TA. 1999 - Bioedit: a user-friendly biological sequence alignment editor and analysis program for Windows 95/98/NT. Nucleic Acids Symposium Series 41, 95-98.

He MQ, Zhao RL, Hyde KD, Begerow D et al. 2019 - Notes, outline and divergence times of Basidiomycota. Fungal Diversity 99(1), 105-367. Doi 10.1007/s13225-019-00435-4

He X, Shi ZJ, Zhao CL. 2020 - Morphological and molecular identification of two new species of Tubulicrinis (Hymenochaetaceae, Hymenochaetales) from southern china. Mycoscience 61(4), 184-189. Doi 10.1016/j.myc.2020.03.008

Himani S, Krishnappa M. 2020 - Xylaria oxyacanthae (Xylariaceae), a new record on Diospyros melanoxylon from India. Studies in Fungi 5(1), 485-490. Doi 10.5943/sif/5/1/29

Hipol RM, Baldelomar JA, Bolinget KC, Solis AFF. 2019 - The soil fungi producing siderophores of Mt. Yangbew, Tawang, La Trinidad, Benguet. Studies in Fungi 4(1), 1-13. Doi 10.5943/sif/4/1/1

Hood IA, Ramsfield TD. 2016 - Armillaria aotearoa species nova. New Zealand Journal of Forestry Science 46(1), 2. Doi 10.1186/s40490-016-0058-y

Huang RX, Luo KY, Zhao CL. 2020 - Phlebia nigrodontea sp. nov. in Meruliaceae (Polyporales) with a black hymenial surface. Phytotaxa 458(3), 195-206. Doi 10.11646/phytotaxa.458.3.2.

Kirk PM, Cannon PF, Minter DW, Stalpers JA. 2008 - Ainsworth \& Bisby's Dictionary of the Fungi, 10th edn. CAB International, Wallingford.

Kumar S, Singh R, Kamal. 2021 - Global diversity and distribution of distoseptosporic micromycete Corynespora Güssow (Corynesporascaceae): An updated checklist with current status. Studies in Fungi 6(1), 1-63. Doi: 10.5943/sif/6/1/1

Liu XF, Shen S, Zhao CL. 2019 - Morphological and molecular identification of a new species of Eichleriella (Auriculariales, Basidiomycota) in China. Phytotaxa 404(6), 245-254. Doi 10.11646/phytotaxa.404.6.3

Luo KY, Ma X, Zhao CL. 2019 - Neofavolus yunnanensis sp. nov. (Polyporales, Basidiomycota) from China: evidence from morphology and DNA sequence data. Phytotaxa 408, 109-116. Doi 10.11646/phytotaxa.408.2.3

Ma X, Zhao CL. 2019 - Crepatura ellipsospora gen. et sp. nov. in Phanerochaetaceae (Polyporales, Basidiomycota) bearing a tuberculate hymenial surface. Mycological Progress 18(6), 785 793. Doi 10.1007/s11557-019-01488-0

M'Barek HN, Arif S, Taidi B, Hajjaj H. 2020 - Consolidated bioethanol production from olive mill waste: Wood-decay fungi from central Morocco as promising decomposition and fermentation biocatalysts. Biotechnology Reports, 28. Doi 10.1016/j.btre.2020.e00541

Miettinen O, Larsson E, Sjokvist E, Larsson KH. 2012 - Comprehensive taxon sampling reveals unaccounted diversity and morphological plasticity in a group of dimitic polypores (Polyporales, Basidiomycota). Cladistics-the International Journal of the Willi Hennig Society 28(3), 251-270. Doi 10.1111/j.1096-0031.2011.00380.x

Miller MA, Holder MT, Vos R, Midford PE et al. 2009 - The CIPRES Portals. CIPRES. http://www.phylo.org/sub_sections/portal. 2009-08-04. (Archived by WebCite at http://www.webcitation.org/5imQ1JeQa). 
Moreau PA, Vila J, Aime MC, Antonin V et al. 2015 - Cibaomyces and Cyptotrama, two new genera for Europe, and an emendation of Rhizomarasmius (Basidiomycota, Physalacriaceae). Mycological Progress 14, 4. Doi 10.1007/s11557-015-1024-4

Nylander JAA. 2004 - MrModeltest v2. Program distributed by the author. Evolutionary Biology Centre, Uppsala University.

Petersen JH. 1996 - Farvekort. The Danish Mycological Society's colour-chart. Foreningen til Svampekundskabens Fremme, Greve.

Pildain MB, Rajchenberg M. 2013 - The phylogenetic position of Postia s.l. (Polyporales, Basidiomycota) from Patagonia, Argentina. Mycologia 105(2), 357-367. Doi 10.3852/12-088

Ronquist F, Huelsenbeck JP. 2003 - MrBayes 3: Bayesian phylogenetic inference under mixed models. Bioinformatics 19, 1572-1574. Doi 10.1093/bioinformatics/btg180

Runnel K, Miettinen O, Lohmus A. 2021 - Polypore fungi as a flagship group to indicate changes in biodiversity - a test case from Estonia. IMA Fungus 12(1).

Doi 10.1186/s43008-020-00050-y

Russell R, Paterson M. 2006 - Ganoderma - A therapeutic fungal biofactory. Phytochemistry 67(18), 1985-2001. Doi 10.1016/j.phytochem.2006.07.004

Sarma VV, Hyde KD. 2018 - Fungal species consortia on Nypa fruticans at Brunei. Studies in Fungi 3(1), 19-26. Doi 10.5943/sif/3/1/3

Shen S, Ma X, Xu TM, Zhao CL. 2018 - Phlebia ailaoshanensis sp. nov. (Polyporales, Basidiomycota) evidenced by morphological characters and phylogenetic analyses. Phytotaxa 373, 184-196. Doi 10.11646/phytotaxa.373.3.2

Shi ZW, Wang XW, Zhou LW, Zhao CL. 2019 - Xylodon kunmingensis sp. nov. (Hymenochaetales, Basidiomycota) from southern China. Mycoscience 60, 184-188. Doi 10.1016/j.myc.2019.02.002

Schoch CL, Robbertse B, Robert V, Vu D et al. 2014 - Finding needles in haystacks: linking scientific names, reference specimens and molecular data for Fungi. Database, 1-21. Doi 10.1093/database/bau061

Swofford DL. 2002 - PAUP*: phylogenetic analysis using parsimony (*and other methods). Version 4.0b10. Sinauer Associates, Massachusetts.

Vinay DS, Ryan EP, Pawelec G, Talib WH et al. 2015 - Immune evasion in cancer: Mechanistic basis and therapeutic strategies. Seminars in Cancer Biology 35, S185-S198. Doi 10.1016/j.semcancer.2015.03.004

Vu D, Groenewald M, De VM, Gehrmann T et al. 2019 - Large-scale generation and analysis of filamentous fungal DNA barcodes boosts coverage for kingdom fungi and reveals thresholds for fungal species and higher taxon delimitation. Studies in Mycology 92, 135-154. Doi 10.1016/j.simyco.2018.05.001

Wang RZ, Yuan ZY, Han CZ, Zhao CL. 2020 - Morphological and molecular identification of a new species of Cinereomyces (Polyporales, Basidiomycota) in southern China. Phytotaxa 459(1), 051-060. Doi 10.11646/phytotaxa.459.1.5

Wang H, Zhao CL. 2021 - Hyphodermella aurantiaca sp. nova. (Polyporales, Basidiomycota) as evidenced by morphological characters and phylogenetic analyses. Annales Botanici Fennici $58,61-68$.

White TJ, Bruns T, Lee S, Taylor J. 1990 - Amplification and direct sequencing of fungal ribosomal RNA genes for phylogenetics. In: Innis MA, Gelfand DH, Sninsky JJ, White TJ (eds), PCR Protocols: a guide to methods and applications. Academic Press, New York, pp. 315-322. Doi 10.1016/B978-0-12-372180-8.50042-1

Willis KJ. (ed.) 2018 - State of the World's Fungi 2018. Report. Royal Botanic Gardens, Kew.

Wu F, Yuan Y, He SH, Bandaraet AR et al. 2015 - Global diversity and taxonomy of the Auricularia auricula-judae complex (Auriculariales, Basidiomycota). Mycological Progress 14, 95. Doi 10.1007/s11557-015-1113-4

Wu SH, Chen CL, Wei CL, Chen YP et al. 2020 - Ganoderma bambusicola sp. nov. (Polyporales, Basidiomycota) from southern Asia. Phytotaxa 456(1), 75-85. 
Doi 10.11646/phytotaxa.456.1.5

Wu YX, Wu JR, Zhao CL. 2021 - Steccherinum tenuissimum and S. xanthum spp. nov. (Polyporales, Basidiomycota): New species from China. PLoS One 16(1), e0244520. Doi 10.1371/journal.pone.0244520

Wu ZQ, Shen S, Luo KY, Wang ZH et al. 2017 - Morphological and molecular identification of a new species of Atraporiella (Polyporales, Basidiomycota) in China. Phytotaxa 332, 31-40. Doi 10.11646/phytotaxa.332.1.3

Wu ZQ, Xu TM, Shen S, Liu XF et al. 2018 - Elaphroporia ailaoshanensis gen. et sp nov in Polyporales (Basidiomycota). MycoKeys 29, 81-95. Doi 10.3897/mycokeys.29.22086

Xu SF, Li XY, Xia J, Liao XK. 2015 - Evaluation on forest health condition in Haikou Forest Farm of Kunming. Journal of Southwest Forestry University 35(2), 68-72.

Xu TM, Liu XF, Chen YH, Zhao CL. 2019 - Rhomboidia wuliangshanensis gen. et sp. nov. from southwestern China. Mycotaxon 134, 649-662. Doi 10.5248/134.649

Xiong XW, Zhou HQ. 2019 - Evaluation of health status of Pinus armandii plantation in central Yunnan plateau: a case study of state-owned Haikou Forest Farm in Kunming. Journal of Jiangsu Forestry Science \& Technology 46(3), 1-5.

Yafetto L. 2018 - Protein enrichment of cassava pulp by solid-state fermentation using Aspergillus niger. Studies in Fungi 3(1), 7-18. Doi 10.5943/sif/3/1/2

Zhang ZH, Wang YT, Tie XR, Chen JL. 2017 - The effectiveness evaluation about natural forest protecti on project construction in Kunming Haikou Forest Farm. Journal of Green Science and Technology 9, 142-146.

Zhao CL, Wu ZQ. 2017 - Ceriporiopsis kunmingensis sp. nov. (Polyporales, Basidiomycota) evidenced by morphological characters and phylogenetic analysis. Mycological Progress 16, 93-100. Doi 10.1007/s11557-016-1259-8

Zong TK, Liu CM, Wu JR, Zhao CL. 2021 - Trechispora daweishanensis and T. xantha spp. nov. (Hydnodontaceae, Trechisporales) found in Yunnan Province of China. Phytotaxa 479(2), 147-159. Doi 10.11646/phytotaxa.479.2.1 